\title{
Infrastructure for Retinal Image Analysis
}

\author{
Behdad Dashtbozorg ${ }^{1 \star}$, Samaneh Abbasi-Sureshjani ${ }^{1}$, Jiong Zhang ${ }^{1}$, \\ Fan Huang ${ }^{1}$, Erik Bekkers ${ }^{1}$, and Bart ter Haar Romeny ${ }^{2,1}$ \\ 1 Department of Biomedical Engineering, Eindhoven University of Technology, \\ Eindhoven, the Netherlands \\ 2 Sino-Dutch School for Biomedical and Information Engineering, Northeastern \\ University, Shenyang, China \\ \{B.Dasht.Bozorg, S.Abbasi@tue.nl, J.Zhang1@tue.nl, F.Huang@tue.nl, \\ E.J.Bekkers@tue.nl, B.M.terhaarRomeny@tue.nl\}@tue.nl
}

\begin{abstract}
This paper introduces a retinal image analysis infrastructure for the automatic assessment of biomarkers related to early signs of diabetes, hypertension and other systemic diseases. The developed application provides several tools, namely normalization, vessel enhancement and segmentation, optic disc and fovea detection, junction detection, bifurcation/crossing discrimination, artery/vein classification and red lesion detection. The pipeline of these methods allows the assessment of important biomarkers characterizing dynamic properties of retinal vessels, such as tortuosity, width, fractal dimension and bifurcation geometry features.
\end{abstract}

Keywords: Retina, biomarkers, diabetes, orientation scores, curvature, vessel segmentation, artery/vein classification, bifurcation, optic disc, fovea, microaneurysm, fractal dimension

\section{Introduction}

Retinal image analysis plays an important role in the detection of early signs of several systemic diseases, namely diabetes, hypertension and other vascular disorders. Automated retinal image analysis offers the possibility of examination of a large number of images with time and cost savings and offers more objective measurements than current observation techniques in a clinical context.

This paper presents a retinal image analysis infrastructure which provides tools for the automated detection and segmentation of important retinal landmarks, as well as for the assessment of vascular changes. Based on this infrastructure, an application has been developed which automatically computes several biomarkers from retinal images in a repeatable and objective manner. The algorithms used in this application outperform most of the state-of-the-art techniques [10].

In Section 2, we introduce the infrastructure and the methodologies for retinal image analysis. Section 3 describes our approach for the assessment of biomarkers, and finally we conclude in Section 4.

\footnotetext{
^ The authors have contributed equally to this work.
} 


\section{Methodologies}

Analysis of retinal biomarkers involves a big range of separate and dependent sequential modules. In Fig. 1 we illustrate how these modules interact with each other. Each of these modules and the computed biomarkers are explained in the following sections.

\subsection{Orientation score transformation}

An orientation score is a 3D data representation Fig. 1(d) in which image data is re-organized on the basis of both positions and orientations. The orientation score representation is highly suited for the analysis of vessels since curvilinear structures are manifestly disentangled according to their local orientations.

The orientation score transform is a wavelet-type transform [8], in which an image is filtered by a set of rotated anisotropic wavelets, i.e. cake wavelets [4]. The set of filter responses forms an orientation score and is regarded as a function on the space of positions and orientations $\mathbb{R}^{2} \rtimes S^{1}$. In the analysis of an orientation score, its domain is identified with the Lie group $\mathbb{R}^{2} \rtimes S^{1} \equiv S E(2)$ : the group of planar translations and rotations.

In this work, we take the full advantages of orientation score and integrate it into different applications, essentially because it provides us with geometric basics for processing image data and analyzing clinical biomarkers in a new perspective. Most of the features such as vascular tree, optic disk and fovea, curvature, crossing/bifurcation and vessel caliber are extracted based on processing orientation scores, as shown in Fig. 1(f).

\subsection{Vessel enhancement and segmentation}

Retinal vascular analysis requires a well-extracted vessel tree from the original image. In the context of large screening programs, we need an efficient and accurate vessel segmentation algorithm to assist ophthalmologists. Here we employ rotating multi-scale Gaussian derivative filters [13] in orientation scores for the enhancement and segmentation of blood vessels. The rotating derivatives are taken in the directions that are perpendicular to the vessel structures at their corresponding orientation planes. Disentangled vessel segments in the 3D orientation scores are enhanced by the rotating filters with proper scale samples, and afterwards the 2D enhanced vessel map is obtained by taking the maximum filter response over all orientation per position. By defining a proper threshold value on the enhanced image, the binary vascular map is finally obtained (Fig. 1(h)). For images taken with the scanning laser ophthalmoscopy (SLO) technology, we use similar multi-scale and multi-orientation features in a supervised manner for enhancing and segmenting the blood vessels [2].

\subsection{Curvature measurement}

Vessel tortuosity is one of the main biomarkers of the retinal vasculature associated to systematic diseases such as diabetes and hypertension. It is measured 


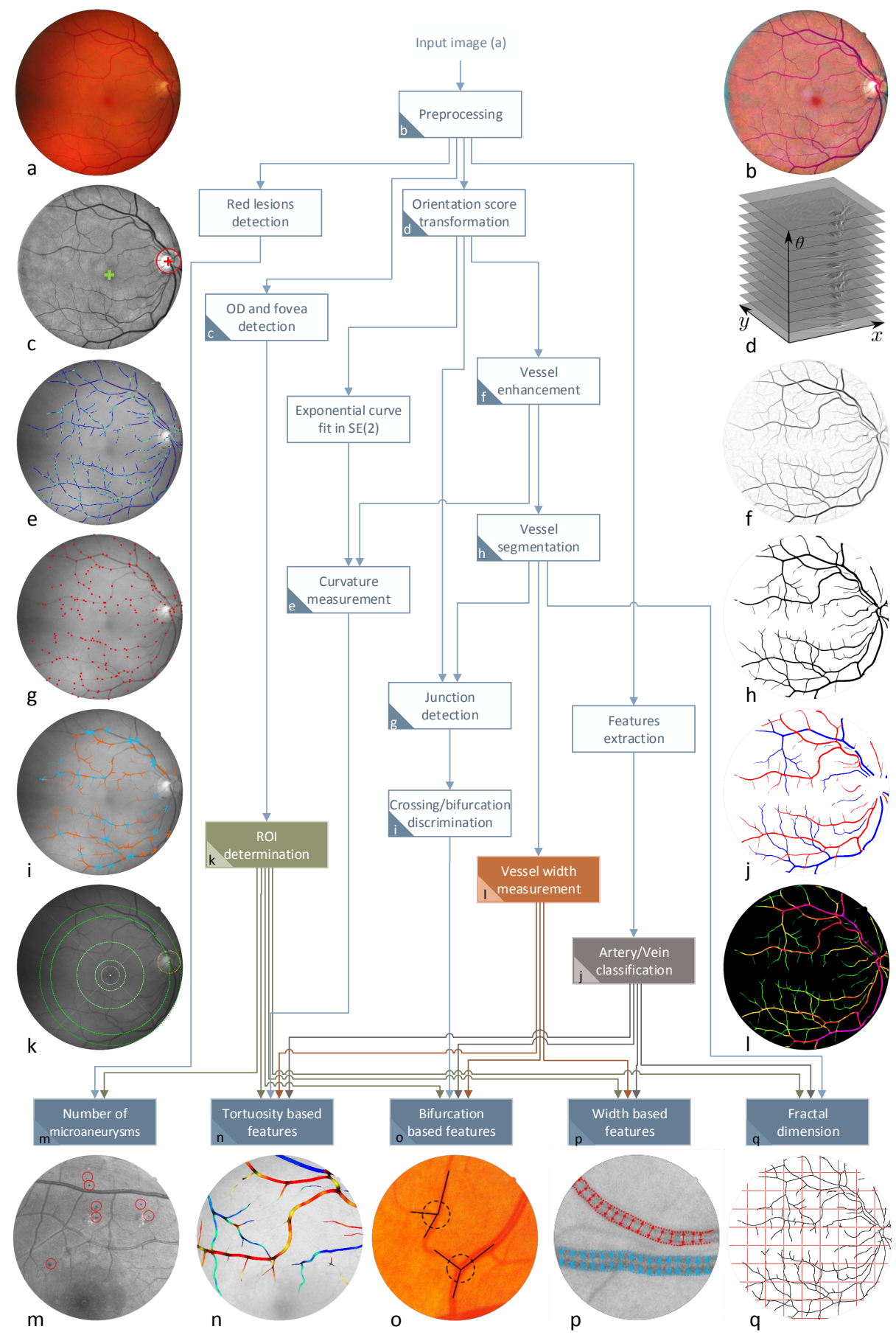

Fig. 1: The infrastructure for retinal image analysis; (a) original fundus image, (b) normalized image, (c) OD and fovea locations, (d) orientation scores, (e) curvature map, (f) vessel probability map, (g) junctions, (h) vessel binary map, (i) bifurcations and crossings, (j) Artery/vein classes, (k) regions of interest, (l) vessel caliber map, (m) microaneurysm detcetion, (n-q) assessment of biomarkers including global tortuosity, bifurcation features, width and fractal dimension. 
based on the local curvature of blood vessels, which indicates the rate of change in orientation, and which might be increased because of diseases. The common practice for measuring the vessel curvature is based on an initial vessel segmentation. By finding the vascular skeleton, splitting the skeleton at junction positions, fitting a curve to each segment and parameterization of these curves, the local curvatures of these segments are obtained. To avoid propagation of errors in each step of such pipelines, a new alternative local curvature extraction method was proposed in [3]. The method relies on the theory of exponential curve fits in orientation scores [7]. In orientation scores exponential curves are circular spirals, which are essentially straight lines with respect to the curved geometry of the $\mathbb{R}^{2} \rtimes S^{1}$ space. The best fit is obtained by eigensystem analysis of the Gaussian Hessian in the lifted space and their spatial projections directly define the curvature at each point (Fig. 1(e)). The obtained 2D curvature maps are filtered in a final stage by the vessel enhancement results (Fig. 1(f)). This includes the vesselness probabilities in final curvature map as well.

\subsection{Bifurcations and crossing detection}

Bifurcations and crossovers are considered as important landmarks in retinal vasculature. Not only the vessel properties at these points provides relevant clinical information [11], but also they are considered as key points in vessel tracking methods, which represent the graphical model of the vasculature.

For the vessel junction detection, we have used the BICROS method described in [1]. In this method, after applying appropriate preprocessing, the image is lifted with anisotropic (single-sided) cake wavelets with 24 orientations as described in Sec. 2.1. Then for each point, by setting an appropriate threshold value and neighbourhood size, a set of local maximums in orientation column $(\theta$ direction) is detected. Using this information, the locations with more than two local maximums are then considered as candidate junctions. Wrong candidates are eliminated in a later stage using several local and contextual features and a neural network classifier. In addition, the performance is improved by introducing a fusion step, in which the orientation score-based junctions are combined with the ones obtained by applying morphological thinning on skeleton of image segmentation (Fig. 1(g)). Finally, the number of dominant orientations for each junction is used to distinguish between bifurcations and crossings (Fig. 1(i)). The method has been validated quantitatively and qualitatively using two public datasets [1].

\subsection{Optic disc and fovea detection}

The optic disc (OD) and fovea locations are required to determine protocolized regions of interest for the assessment of signs related to vascular changes, such as fractal dimension, tortuosity, central retinal artery/vein equivalent and central artery-to-vein diameter ratio. On the other hand, the macula is essential for sharp central vision, therefore the locations of lesions, such as microaneurysms, 
hemorrhages and drusen with respect to the center of macula (fovea) are important for disease classification and grading.

In this work, we have used the automatic OD and fovea detection technique proposed in [5], where a new convergence index operator, called super-elliptical filter (SEF) is presented. The SEF is suitable for the detection of semi-elliptical convex shapes and as such it performs well for the OD localization. Furthermore, a setup for the simultaneous localization of the OD and fovea is introduced, in which the detection result of one landmark facilitates the detection of the other one. This design, called PSEF, contains two individual SEF filters which are located within a specific distance of each other. The distance between the SEF filters is constrained in accordance to the average of vertical and horizontal distances between the OD and the fovea in the human eye. The PSEF filter is applied on the normalized green channel image (Fig. 1(b)) and then the locations of $\mathrm{OD}$ and fovea are obtained by finding the coordinates corresponding to the maximum filter response as shown in Fig. 1(c).

\subsection{Artery/vein classification}

The vasculature on the human retina can be divided into arteries and veins. Arteries and veins behave differently under pathological conditions. As such, it is important to define and study biomarkers separately for arteries and veins.

In this work, we have used a supervised technique to classify the vessels into arteries and veins. Firstly, we obtain the vessel pixels from the vessel binary map described in Sec. 2.2. Then for each pixel, we extract in total 455 features including, among others: the local intensity of the RGB and HSB color channels; the mean, standard deviation, median, minimum and maximum of the intensities inside small, medium and large circular regions; the intensity values along each vessel centerline; and the intensity inside each vessel segment. After that, a genetic-based feature selection approach proposed in [9], is used to select the subset of features giving the highest classification accuracy from the 455 features. Finally, a logistic regression classifier and the set of selected features are used for the classification of arteries and veins.

\section{Biomarkers}

In order to standardize the biomarkers, all values are calculated from the vessels inside specified regions of interest (ROI) around the fovea or optic disc (OD) centers (Fig. 1(k)). For the fovea centered images, the regions are defined by disks centered at the fovea location with the radii of 3,5 and 6 times the OD radius $\left(r_{O D}\right)$, while for the OD centered images, the regions are specified as the standard ring areas within 2 to 3,5 or $6 r_{O D}$ from the OD center. The $r_{O D}$ is set in accordance to the average of optic disc radius in the human eye. 


\subsection{Vessel width biomarkers}

Vessel width is one important biomarker in the clinical study. The change of vessel caliber directly reflects the change of blood flow viscosity and blood pressure in the vessels. In this work, we measure the central retinal arterial equivalent (CRAE), central retinal venous equivalent (CRVE) and the arterial-venous ratio (AVR) of the retinal vasculature in three different regions of interest. Firstly, the vessels within the defined ROI are selected. Then for each segment, the vessel caliber is obtained by using a distance transform technique. Finally, an approach similar to the one described in [6] is applied for the estimation of CRAE, CRVE and AVR within different ROIs.

\subsection{Global tortuosity biomarkers}

For the assessment of biomarkers indicating the global tortuosity, the local curvature values are extracted from the curvature maps (see Sec. 2.3) for the vessel pixels obtained in Sec. 2.2 (Fig. 1(n)). Afterwards, several statistical descriptors such as average, median and standard deviation of the entire distribution of curvature values from a single image are calculated within the defined ROI. The global tortuosity biomarkers are computed for different groups of vessels based on their caliber (small, medium and large vessels), and their type (artery and vein).

\subsection{Bifurcation biomarkers}

In order to investigate the geometric properties at bifurcation points detected in Fig. 1(i), several features introduced by [11] have been calculated. These features include bifurcation angles, the asymmetry, area and diameter ratios, the bifurcation index and optimality parameters. It is essential to measure vessel widths (Fig. 1(l)) and determine the artery/vein labels (Fig. 1(j)) for a specific ROI (Fig. 1(k)) beforehand. By having this prerequisite information, all these features are calculated for arteries and vein separately and their mean, standard deviation, median, minimum and maximum are found for each vessel type.

\subsection{Fractal dimension and lacunarity biomarkers}

Another biomarker that has been studied for many years is the fractal dimension. The vascular tree on the human retina is a fractal object, because similar branching patterns appear among different scales. Fractal dimension is a measure of the complexity of a fractal object. It has been proposed in the literature that fractal dimension is related to the progress of DR and other vascular diseases [12]. In this work, we estimate the fractal dimension of a vascular network by using the box, information, correlation and the multi-fractal dimensions and the lacunarity (Fig. 1(o)). The fractal dimensions are not only computed on the full vascular network, but also on the arterial and venous network separately, using the result of artery/vein separation introduced in Sec. 2.6. 

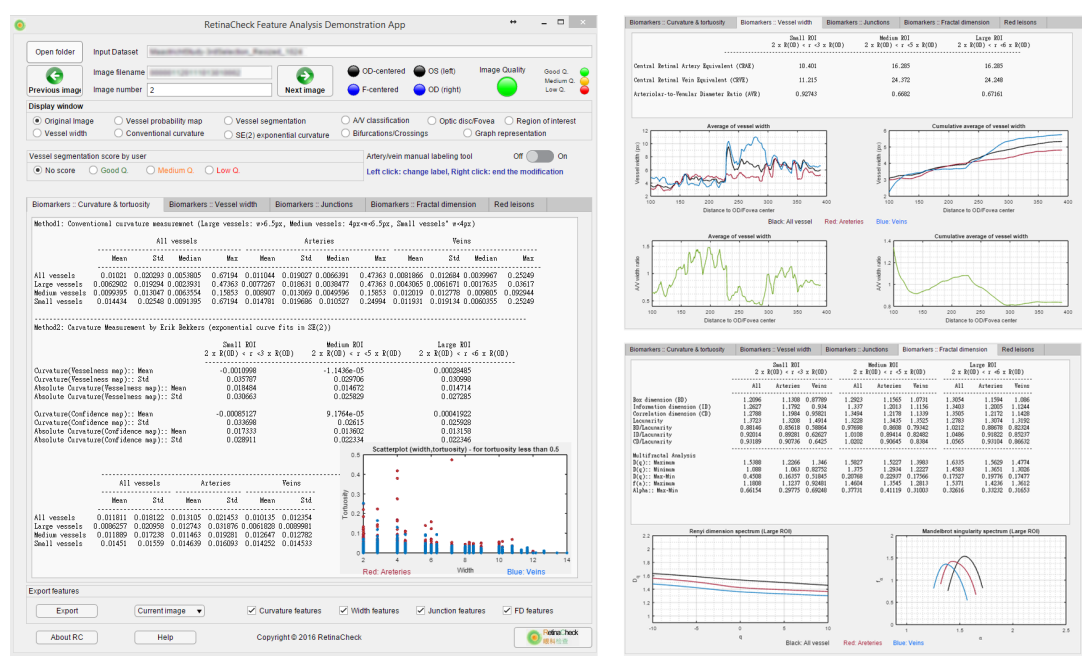

Fig. 2: The Interface of retinal image analysis application and generated reports

\subsection{Microaneurysm counts}

Microaneurysms (MAs) are among the early signs of DR. They are small swellings which are caused by a weakening of the vessel wall and located at the side of tiny blood vessels. In digital color fundus images, MAs appear as tiny, reddish isolated dots. For the detection of MAs, a supervised classification technique is used to discriminate between MAs and other structures like background textures. After applying a multi-scale and multi-orientation gradient weighting method on a normalized image, the small components including the MAs will appear in the form of doughnut shaped structures. Afterwards, a set of candidates is obtained using a recursive thresholding procedure, where the candidates are constrained by considering the properties of the doughnut shaped structures. Then the MAs are detected using the RUSBoost classifier and a set of features including the local intensity, the shape features and the responses of convergence index filters. Finally, the numbers of MAs in foveal, parafovea and prefoveal regions are recorded as biomarkers (Fig. 1(m)).

\section{Conclusion}

The paper presents an infrastructure for retinal image analysis and gives a review of several developed tools with the goal of detecting early signs of several systematic diseases. Based on this infrastructure, a dedicated application for the automated assessment of retinal health biomarkers has been developed. Fig. 2 shows the main interface of this application and the automated generated reports. The validation phase is ongoing and the application is currently being tested in Maastricht University Eye Hospital, on over 10,000 subjects and patients with a broad spectrum of clinical measurements. 
Acknowledgments. The work is part of the Hé Programme of Innovation Cooperation, which is financed by the Netherlands Organization for Scientific Research (NWO), dossier No. 629.001.003.

\section{References}

1. Abbasi-Sureshjani, S., Smit-Ockeloen, I., Bekkers, E., Dashtbozorg, B., ter Haar Romeny, B.: Automatic detection of vascular bifurcations and crossings in retinal images using orientation scores. In: 2016 IEEE 13th International Symposium on Biomedical Imaging (ISBI). pp. 189-192 (2016)

2. Abbasi-Sureshjani, S., Smit-Ockeloen, I., Zhang, J., Ter Haar Romeny, B.: Biologically-inspired supervised vasculature segmentation in slo retinal fundus images. In: ICIAR 2015, LNCS. pp. 325-334. Springer, Heidelberg (2015)

3. Bekkers, E., Zhang, J., Duits, R., ter Haar Romeny, B.: Curvature based biomarkers for diabetic retinopathy via exponential curve fits in $\mathrm{SE}(2)$. In: Proceedings of the Ophthalmic Medical Image Analysis Second International Workshop, OMIA 2015 Held in Conjunction with MICCAI, 2015. pp. 113-120 (2015)

4. Bekkers, E., Duits, R., Berendschot, T., ter Haar Romeny, B.: A multi-orientation analysis approach to retinal vessel tracking. Journal of Mathematical Imaging and Vision 49(3), 583-610 (2014)

5. Dashtbozorg, B., Zhang, J., Huang, F., ter Haar Romeny, B.: Automatic optic disc and fovea detection in retinal images using super-elliptical convergence index filters. In: ICIAR 2016, LNCS. vol. 9730, pp. 1-10. Springer, Heidelberg (2016)

6. Dashtzbozorg, B., Mendonca, M, A., Penas, S., Campilho, A.: Computer-aided diagnosis system for the assessment of retinal vascular changes. In: Proceedings of the Ophthalmic Medical Image Analysis First International Workshop, OMIA 2014, Held in Conjunction with MICCAI 2014. pp. 9-16 (2014)

7. Duits, R., Janssen, M., Hannink, J., Sanguinetti, G.: Locally adaptive frames in the roto-translation group and their applications in medical imaging. Journal of Mathematical Imaging and Vision pp. 1-36 (2016)

8. Duits, R., Felsberg, M., Granlund, G., ter Haar Romeny, B.: Image analysis and reconstruction using a wavelet transform constructed from a reducible representation of the euclidean motion group. International Journal of Computer Vision 72(1), 79-102 (2007)

9. Eiben, A.E., Smith, J.E.: Introduction to evolutionary computing. Springer, second edn. (2015)

10. ter Haar Romeny, B., Bekkers, E., Zhang, J., Abbasi-Sureshjani, S., Huang, F., Duits, R., Dashtbozorg, B., Berendschot, T., et al.: Brain-inspired algorithms for retinal image analysis. Machine Vision and Applications pp. 1-19 (2016)

11. Habib, M., Al-Diri, B., Hunter, A., Steel, D.: The association between retinal vascular geometry changes and diabetic retinopathy and their role in prediction of progression-an exploratory study. BMC Ophthalmology 14(1), 89 (2014)

12. Huang, F., Zhang, J., Bekkers, E., Dashtbozorg, B., ter Haar Romeny, B.: Stability analysis of fractal dimension in retinal vasculature. In: Proceedings of the Ophthalmic Medical Image Analysis Second International Workshop, OMIA 2015 Held in Conjunction with MICCAI 2015. pp. 1-8 (2015)

13. Zhang, J., Bekkers, E., Abbasi-Sureshjani, S., Dashtbozorg, B., ter Haar Romeny, B.: Robust and fast vessel segmentation via Gaussian derivatives in orientation scores. In: ICIAP 2015, LNCS. vol. 9279, pp. 537-547. Springer, Heidelberg (2015) 